\title{
Outcomes of Cushing's disease following Gamma Knife radiosurgery: effect of a center's growing experience and era of treatment
}

\author{
Adomas Bunevicius, MD, PhD, ${ }^{1}$ Darrah Sheehan, ${ }^{1}$ Mary Lee Vance, MD, ${ }^{1,2}$ David Schlesinger, PhD, ${ }^{1}$ \\ and Jason P. Sheehan, MD, PhD'
}

Departments of ${ }^{1}$ Neurological Surgery and ${ }^{2}$ Medicine, University of Virginia Health System, Charlottesville, Virginia

OBJECTIVE Stereotactic radiosurgery (SRS) is used for the management of residual or recurrent Cushing's disease (CD). Increasing experience and technological advancements of Gamma Knife radiosurgery (GKRS) systems can impact the outcomes of $C D$ patients. The authors evaluated the association of their center's growing experience and the era in which GKRS was performed with treatment success and adverse events in patients with $C D$.

METHODS The authors studied consecutive patients with CD treated with GKRS at the University of Virginia since installation of the first Gamma Knife system in March 1989 through August 2019. They compared endocrine remission and complication rates between patients treated before 2000 (early cohort) and those who were treated in 2000 and later (contemporary cohort).

RESULTS One hundred thirty-four patients with CD underwent GKRS during the study period: 55 patients (41\%) comprised the early cohort, and 79 patients (59\%) comprised the contemporary cohort. The contemporary cohort, compared with the early cohort, had a significantly greater treatment volume, radiation prescription dose, maximal dose to the optic chiasm, and number of isocenters, and they more often had cavernous sinus involvement. Endocrine remission rates were higher in the contemporary cohort when compared with the early cohort ( $82 \%$ vs $66 \%$, respectively; $p=0.01$ ). In a Cox regression analysis adjusted for demographic, clinical, and SRS characteristics, the contemporary GKRS cohort had a higher probability of endocrine remission than the early cohort (HR 1.987, $95 \% \mathrm{Cl} 1.234-3.199 ; p=0.005)$. The tumor control rate, incidence of cranial nerve neuropathy, and new anterior pituitary deficiency were similar between the two groups.

CONCLUSIONS Technological advancements over the years and growing center experience were important factors for improved endocrine remission rates in patients with CD. Technological aspects and results of contemporary Gamma Knife systems should be considered when counseling patients, planning treatment, and reporting treatment results. Studies exploring the learning curve for GKRS are warranted.

https://thejns.org/doi/abs/10.3171/2019.12.JNS192743

KEYWORDS Cushing's disease; stereotactic radiosurgery; Gamma Knife; prognosis; remission; pituitary surgery

$\mathrm{C}$ USHING's disease (CD) is a rare disorder caused by autonomous secretion of adrenocorticotropic hormone (ACTH) by a pituitary adenoma that results in cortisol hypersecretion by the adrenal glands. Common symptoms of CD include central obesity, diabetes, hypertension, mood changes, and accelerated cognitive decline ${ }^{5,28} \mathrm{CD}$ patients are at up to a fivefold increased mortality risk. ${ }^{3,5,13}$ Normalization of hypercortisolemia is the treatment goal, because prolonged hypercortisolemia,,$^{10}$ persistent hypercortisolemia after pituitary adenoma resection, ${ }^{7}$ and higher number of prior treatments ${ }^{3}$ increase the risk for unfavorable outcomes.

The management of $\mathrm{CD}$ often requires multidisciplinary expertise that includes a neurosurgeon, endocrinologist, and often a radiation oncologist and medical physicist. Surgical resection of an ACTH-producing pituitary

ABBREVIATION ACTH = adrenocorticotropic hormone; $A R E$ = adverse radiation event; $C D$ = Cushing's disease; GKRS = Gamma Knife radiosurgery; SRS = stereotactic radiosurgery; UFC = urinary free cortisol.

SUBMITTED October 8, 2019. ACCEPTED December 2, 2019.

INCLUDE WHEN CITING Published online January 31, 2020; DOI: 10.3171/2019.12.JNS192743. 
adenoma is the central treatment of CD..$^{21,23,31}$ However, in up to $35 \%$ of CD patients, remission after adenoma resection either fails to occur or tumor recurrence develops. ${ }^{25,26}$ An important limiting factor influencing surgical remission is infiltration of the cavernous sinus by the adenoma. Stereotactic radiosurgery (SRS) is used when endocrine remission cannot be achieved after failed pituitary surgery, with recurrence, or when a patient is not deemed fit for or refuses surgical treatment. ${ }^{21}$ SRS is generally a safe treatment method that results in a durable endocrine remission in up to $80 \%$ of CD patients. ${ }^{19}$

The Gamma Knife (Elekta) was invented in the 1950s by Dr. Lars Leksell. ${ }^{12}$ The first commercially available Gamma Knife radiosurgery (GKRS) system in the US was installed in 1987.32 GKRS was accepted and became an important part of treatments in the management of a spectrum intracranial lesions, including incompletely resected or recurrent ACTH-producing pituitary adenomas. While the principles of GKRS remain largely unchanged, there have been numerous advances in the GKRS systems and planning software throughout its evolution, including (but not limited to) an ability to deliver multiple isocenter plans, ability to use head CT and subsequently stereotactic MRI for treatment planning, introduction of an automated patient positioning system, as well as more efficient, user-friendly, and precise radiosurgery treatment planning software. . $^{6,9,14,20,29}$ These innovations improved the accuracy and precision of GKRS targeting and delivery. However, the association between these technological advancements of GKRS with treatment outcomes remains largely unstudied.

Increasing experience of a center with GKRS and other surgical and medical management options of CD are also important for optimal patient outcomes. Greater experience and the caseload of a neurosurgeon and a center are important predictors of better outcomes and lower complication rates across neurosurgical procedures, including transsphenoidal resection of pituitary adenomas, ${ }^{4}$ excision of vestibular schwannomas, ${ }^{2,18}$ the clipping ${ }^{11}$ and coiling ${ }^{1}$ of intracranial aneurysms, and scoliosis correction surgery, ${ }^{8}$ among others. However, to the best of our knowledge, there are no studies evaluating the possible association of experience with GKRS and treatment outcomes of patients with $\mathrm{CD}$.

The goal of this study was to evaluate the possible association of the era of GKRS (as a proxy of the center's experience and technological advancements) with treatment success and the risk adverse events of patients with CD. We hypothesized that because of technological advances and increasing center experience, patient outcomes would improve and the risk adverse events would decrease in patients treated at later time points compared with patients treated earlier.

\section{Methods \\ Patients}

The data were collected retrospectively with approval from the Institutional Review Board of the University of Virginia. Because of the retrospective study design, informed consents specific to the data collection for this study were not obtained from patients included in the report.

All patients diagnosed with ACTH-dependent CD and treated with GKRS at the University of Virginia since the inception of GKRS in 1989 up to August 2019 were identified from the institutional database. We considered only patients who were diagnosed with $\mathrm{CD}$ according to the existing guidelines. Also, patients were required to have at least one follow-up evaluation that should have included endocrine testing and neuroimaging after GKRS to be included in the study. Individual patient data were de-identified and pooled for the analyses.

Data from $134 \mathrm{CD}$ patients who met the study inclusion criteria were analyzed.

\section{Patient Evaluation}

The diagnosis of $\mathrm{CD}$ was established according to existing guidelines and included measurement of serum cortisol, ACTH, and 24-hour urinary free cortisol (UFC), as well as other tests as deemed appropriate. ${ }^{19}$ All patients also had radiological confirmation of a pituitary adenoma and endocrine confirmation of an ACTH-secreting pituitary adenoma. Patients who underwent pituitary adenoma resection also had pathological confirmation of an ACTH-producing adenoma. Prior to radiosurgery and at follow-up visits, patients were subjected to neurological, endocrinological, and ophthalmological assessments, as indicated. Patient demographic information and prior treatment details were also recorded.

\section{Radiosurgical Approach}

Single-session GKRS was performed using Gamma Knife unit models U, C, Perfexion, and Icon (Elekta AB). The first Leksell Gamma Unit at the University of Virginia was installed in March 1989. Leksell Gamma Knife model $\mathrm{C}$ was installed in 2001, Perfexion in 2007, and Icon in 2016. Radiosurgical techniques have been described in detail previously. ${ }^{19}$ In brief, SRS was performed with frame-based stereotaxy using the Leksell Model G frame (Elekta AB); the patient was placed under local anesthesia with conscious sedation. Initially, stereotactic CT scanning was used for treatment planning, but around the year 2000, high-resolution MRI became the standard of care. Currently, radiosurgical imaging typically includes highresolution 1-mm-thick pre- and postcontrast T1-weighted 3-T MRI scans. GKRS dose planning was performed by a multidisciplinary team that included a neurosurgeon, radiation oncologist, and medical physicist. Planning was tailored toward individual patient needs and imaging findings; however, the maximal radiation dose to the optic nerve, chiasms, and tracts was generally kept below 8 to 12 Gy. Radiosurgical parameters, including the margin and maximum dose, optic apparatus maximal dose, the isodose line, the tumor volume, and the number of isocenters were recorded in all cases.

\section{Clinical and Radiological Follow-Up}

Follow-up typically included 24-hour UFC (off medication affecting cortisol production or action) and MRI at 6-month intervals for the first 2 years and annually 
thereafter. Endocrine remission was defined as normalization of UFC or morning serum cortisol (as determined by institutional reference ranges) while off of medication to control hypercortisolism. The interval between GKRS and endocrine remission was recorded in all patients. Endocrine recurrence was defined as increase of 24-hour UFC or serum morning cortisol concentration exceeding the reference ranges.

All patients were monitored with serial brain MRI or CT scanning. Tumor progression was defined as an increase of adenoma volume by $20 \%$ on the most recent brain MR images when compared with adenoma size on the pre-GKRS MR image or CT scan..$^{30}$ The interval from GKRS to the last brain MR image was recorded in all patients.

All patients were carefully monitored for adverse radiation events (AREs), including visual complications and dysfunction of other hypothalamic-pituitary axes. Visual field deficits were identified during clinical examination and confirmed by formal visual field testing. Other cranial nerve neuropathies were also documented. Pituitary insufficiency was defined as a new hormone deficit(s) after GKRS. Other treatments during the follow-up period, including repeat GKRS, pituitary adenoma surgery, and bilateral adrenalectomy, were recorded.

\section{Statistical Analysis}

Statistical analyses were performed with SPSS Statistics for Windows software (version 25.0; IBM Corp.). For all statistical tests, a p value $<0.05$ was considered as statistically significant. Categorical data were compared using the Pearson chi-square test, and continuous data were compared using ANOVA.

All patients were stratified into two cohorts based on the year of GKRS: 1) before the year 2000 and 2) year 2000 or later. Kaplan-Meyer analysis with log-rank testing was performed to analyze the association of the GKRS era (in or after 2000 vs before 2000) with time to endocrine remission. A univariate Cox-regression analysis was performed to evaluate the association between the GKRS era (in or after 2000 vs before 2000) with endocrine remission rates at 6 months, 1 year, 2 years, 5 years, and at last endocrine follow-up after SRS. Significant association in univariate Cox regression analyses were adjusted for patient age (years), sex, pre-GKRS surgery (yes or no), pre-GKRS fractionated radiation therapy (yes or no), volume treated (in cubic centimeters $\left[\mathrm{cm}^{3}\right]$ ), prescription dose (Gy), treatment of the whole sella and cavernous sinuses (yes or no). Categorical variables were compared using the Pearson chi-square test and continuous variables with the one-way ANOVA. The results of Cox-regression analysis are presented as hazard ratios, $95 \%$ confidence intervals, and $\mathrm{p}$ values.

\section{Results \\ Patient Characteristics}

One hundred thirty-four patients underwent GKRS for CD between 1990 and August 2019. The number of patients treated each year ranged from 0 to 17 . The mean age of the patients at the time of GKRS was $40.9 \pm 12.7$ years (range 12-72 years), and the majority (77\%) of patients were women (Table 1). Fifty-five patients $(41 \%)$ were treated before 2000 and $79(59 \%)$ patients were treated in 2000 and later. Patients treated before 2000 were more likely to have undergone prior radiation therapy when compared with patients treated in 2000 and later (9\% vs $1 \%$, respectively; $\mathrm{p}=0.03$ ). Pituitary deficiencies preceding GKRS were more common in patients treated in 2000 and later than in patients treated before 2000 (42\% and $20 \%$, respectively; $\mathrm{p}=0.008$ ). Patient age, sex, indication for GKRS, histories of pre-GKRS surgery/medical therapy, and visual dysfunction rates were similar between the two groups.

\section{Radiosurgical Parameters}

SRS procedural parameters in the two groups of patients are presented in Table 2. Patients treated in 2000 or later, when compared with patients treated before 2000, had larger treatment volume $\left(3.27 \pm 1.51 \mathrm{~cm}^{3}\right.$ vs $1.32 \pm$ $\left.1.00 \mathrm{~cm}^{3} ; \mathrm{p}<0.001\right)$, higher radiation prescription dose $(23.07 \pm 2.53$ Gy vs $20.30 \pm 5.77$ Gy; $p<0.001)$, and a greater number of isocenters per radiosurgical procedure $(10.71 \pm 4.21$ vs $4.58 \pm 2.49 ; \mathrm{p}<0.001)$. The cavernous sinus was more often included in the treatment plan in patients treated after $2000(\mathrm{p}<0.001)$, while the suprasellar component was more often targeted before 2000 ( $p<$ 0.01 ). The maximal dose to the optic chiasm was greater in patients treated in the later GKRS group than patients treated earlier $(6.36 \pm 2.18$ Gy and $3.32 \pm 2.31 \mathrm{~Gy} ; \mathrm{p}<$ $0.001)$.

\section{Endocrine Remission}

The mean duration of endocrine follow-up in the overall population was $63.8 \pm 46.8$ months (range 6-210 months). As expected, the duration of endocrine follow-up was longer in the early GKRS group than the late GKRS group $(81.8 \pm 57.4$ months vs $51.3 \pm 32.7$ months; $\mathrm{p}<0.001)$. Kaplan-Meier analyses showed a significant association between the GKRS era and endocrine remission rates (logrank test $=11.069, p=0.01$; Fig. 1). Specifically, endocrine remission rates at 1,2 , and 5 years and at last follow-up were higher in patients treated with GKRS in year 2000 and beyond (44\%, 63\%, $82 \%$, and $82 \%$, respectively) than in patients treated with GKRS before 2000 (16\%, 38\%, $64 \%$, and $66 \%$, respectively).

In univariate Cox regression analyses, patients who underwent GKRS in 2000 or later had a significantly greater probability of endocrine remission at 6 months, 2 years, 5 years, and at last follow-up when compared with patients who underwent GKRS before the year 2000 (Table 3). After adjusting for patient age, sex, pre-GKRS surgery, preGKRS fractionated radiation therapy, prescription dose (Gy), treatment volume $\left(\mathrm{cm}^{3}\right)$, treatment of the sella, suprasellar area, and cavernous sinus, late GKRS was associated with greater probability of endocrine remission relative to early GKRS at the 1-year follow-up (HR 2.259, 95\% CI 1.219-4.186; $\mathrm{p}=0.01$ ), 2-year follow-up (HR 2.501, 95\% CI 1.448-4.320; $\mathrm{p}=0.001$ ), 5-year follow-up (HR 2.021, 95\% CI 1.249-3.268; $\mathrm{p}=0.004)$, and during the entire study period (HR 1.987, 95\% CI 1.234-3.199; $\mathrm{p}=0.005$; 
TABLE 1. Baseline clinical and demographic characteristics as a function of radiosurgery era

\begin{tabular}{|c|c|c|c|c|}
\hline \multirow[b]{2}{*}{ Characteristic } & \multirow[b]{2}{*}{ Total Sample $(n=134)$} & \multicolumn{2}{|c|}{ Era of GKRS } & \multirow{2}{*}{$\begin{array}{l}\text { Chi-Square }(p) \text { or } \\
\text { ANOVA F-Value }(p)\end{array}$} \\
\hline & & Before $2000(n=55)$ & Btwn $2000 \& 2019(n=79)$ & \\
\hline Sex & & & & $0.282(0.595)$ \\
\hline Men & $31(23 \%)$ & $14(26 \%)$ & $17(22 \%)$ & \\
\hline Women & $103(77 \%)$ & $41(74 \%)$ & $62(78 \%)$ & \\
\hline Age at GKRS, yrs & $40.9 \pm 12.7$ & $38.7 \pm 12.2$ & $42.5 \pm 13.0$ & $2.963(0.09)$ \\
\hline Radiosurgery indications & & & & $2.916(0.09)$ \\
\hline Residual & $2(2 \%)$ & $2(4 \%)$ & $0(0 \%)$ & \\
\hline Recurrent & $132(98 \%)$ & $53(96 \%)$ & $79(100 \%)$ & \\
\hline Pre-GKRS transsphenoidal resection & & & & $2.916(0.09)$ \\
\hline Yes & $132(98 \%)$ & $53(96 \%)$ & $79(100 \%)$ & \\
\hline No & $2(2 \%)$ & $2(2 \%)$ & $0(0 \%)$ & \\
\hline Pre-GKRS craniotomy & & & & $0.833(0.362)$ \\
\hline Yes & $3(2 \%)$ & $2(4 \%)$ & $1(1 \%)$ & \\
\hline No & $131(98 \%)$ & $53(96 \%)$ & $78(99 \%)$ & \\
\hline Pre-GKRS fractionated radiation therapy & & & & $4.642(0.031)$ \\
\hline Yes & $6(5 \%)$ & $5(9 \%)$ & $1(1 \%)$ & \\
\hline No & $128(96 \%)$ & $50(91 \%)$ & $78(99 \%)$ & \\
\hline Pre-GKRS medical therapy & & & & $1.393(0.238)$ \\
\hline Yes & $32(24 \%)$ & $16(29 \%)$ & $16(20 \%)$ & \\
\hline No & $102(76 \%)$ & $39(71 \%)$ & $63(80 \%)$ & \\
\hline \multicolumn{5}{|l|}{ Pituitary deficiency before GKRS } \\
\hline Any & $44(33 \%)$ & $11(20 \%)$ & $33(42 \%)$ & $6.970(0.008)$ \\
\hline Hypothyroidism & $30(22 \%)$ & $8(15 \%)$ & $22(28 \%)$ & $3.302(0.069)$ \\
\hline Estrogen/testosterone deficiency & $13(10 \%)$ & $1(2 \%)$ & $12(15 \%)$ & $6.618(0.01)$ \\
\hline Growth hormone deficiency & $6(5 \%)$ & $2(4 \%)$ & $4(5 \%)$ & $0.154(0.694)$ \\
\hline Diabetes insipidus & $10(8 \%)$ & $0(0 \%)$ & $10(13 \%)$ & $7.523(0.006)$ \\
\hline Visual deficits before GKRS & & & & $4.119(0.127)$ \\
\hline None & $125(93 \%)$ & $50(91 \%)$ & $75(94 \%)$ & \\
\hline Visual field deficit & $7(5 \%)$ & $5(9 \%)$ & $2(3 \%)$ & \\
\hline Diplopia & $2(2 \%)$ & $0(0 \%)$ & $2(3 \%)$ & \\
\hline Pretreatment 24-hr UFC, $\mu g^{*}$ & $281.9 \pm 921.0$ & $358.9 \pm 1287.5$ & $206.5 \pm 240.4$ & $0.745(0.390)$ \\
\hline Time btwn resection \& GKRS, mos, mean \pm SD & $15.1 \pm 25.7$ & $15.3 \pm 25.5$ & $15.0 \pm 25.9$ & $0.003(0.96)$ \\
\hline Endocrine follow-up, mos & $63.8 \pm 46.8$ & $81.8 \pm 57.4$ & $51.3 \pm 32.7$ & $15.201(<0.001)$ \\
\hline
\end{tabular}

Values are presented as the number (\%) or as the mean \pm SD. Boldface type indicates statistical significance.

${ }^{*}$ Available for 109 patients.

TABLE 2. Tumor and treatment characteristics as a function of treatment time after surgery

\begin{tabular}{|c|c|c|c|c|}
\hline \multirow[b]{2}{*}{ Characteristics } & \multirow[b]{2}{*}{ Total Sample } & \multicolumn{2}{|c|}{ Era of GKRS } & \multirow{2}{*}{$\begin{array}{c}\text { Chi-Square Test }(p) \text { or ANOVA } \\
\text { F-Value }(p)\end{array}$} \\
\hline & & $<2000$ & $2000-2019$ & \\
\hline Tumor not seen on pre-GKRS imaging & $27(20 \%)$ & $1(2 \%)$ & $26(33 \%)$ & $19.484(<0.001)$ \\
\hline Volume treated, $\mathrm{cm}^{3 *}$ & $2.58 \pm 1.64$ & $1.32 \pm 1.00$ & $3.27 \pm 1.51$ & $52.291(<0.001)$ \\
\hline Cavernous sinus targeted & $92(69 \%)$ & $22(40 \%)$ & $70(89 \%)$ & $35.601(<0.001)$ \\
\hline Suprasellar component targeted & $23(17 \%)$ & $15(27 \%)$ & $8(10 \%)$ & $6.705(0.01)$ \\
\hline Whole sella targeted & $35(26 \%)$ & $16(29 \%)$ & $19(24 \%)$ & $0.427(0.514)$ \\
\hline Margin dose, Gy & $21.93 \pm 4.34$ & $20.30 \pm 5.77$ & $23.07 \pm 2.53$ & $14.246(<0.001)$ \\
\hline Maximal dose, Gy & $46.42 \pm 7.64$ & $46.49 \pm 10.27$ & $46.37 \pm 5.14$ & $0.009(0.93)$ \\
\hline Isodose line, \% & $47.49 \pm 6.65$ & $44.09 \pm 9.31$ & $49.85 \pm 1.30$ & $29.458(<0.001)$ \\
\hline No. of isocenters & $8.19 \pm 4.70$ & $4.58 \pm 2.49$ & $10.71 \pm 4.21$ & $93.435(<0.001)$ \\
\hline Maximal dose to optic chiasm, Gy† & $5.07 \pm 2.69$ & $3.32 \pm 2.31$ & $6.36 \pm 2.18$ & $58.861(<0.001)$ \\
\hline
\end{tabular}




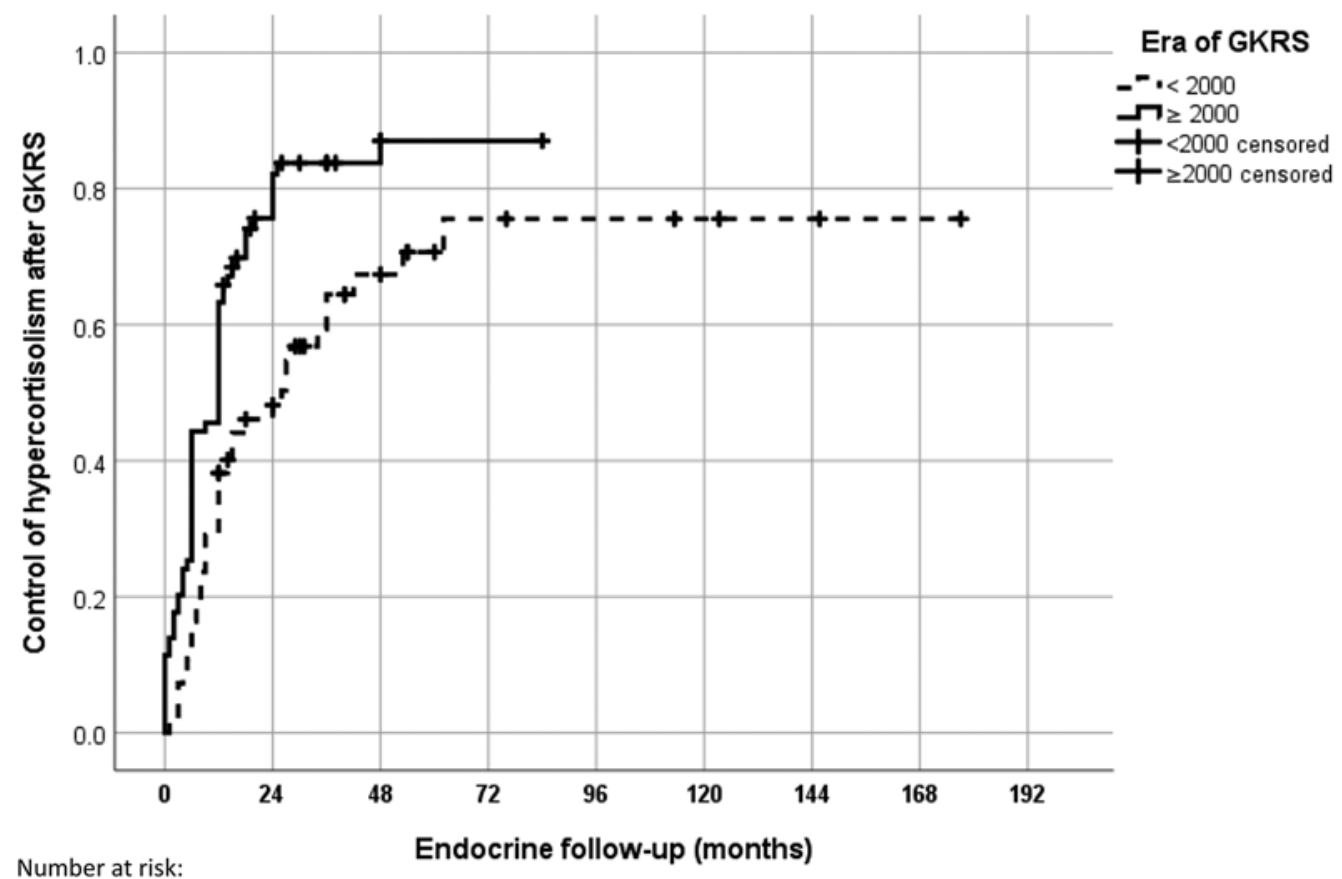

Number at risk:

$\begin{array}{lccccccccc}<2000 & 55 & 26 & 10 & 5 & 4 & 3 & 2 & 1 & 0 \\ \geq 2000 & 79 & 15 & 5 & 1 & 0 & 0 & 0 & 0 & 0\end{array}$

FIG. 1. Association of GKRS era with endocrine remission. Log-rank test $=11.069, p=0.01$.

Table 3). Endocrine recurrence was significantly higher in the early group versus late group ( $36 \%$ vs $8 \%$, respectively; $\mathrm{p}<0.001$; Table 4).

\section{Imaging Outcomes and Adverse Events}

The mean duration of imaging follow-up in the entire cohort was $52.9 \pm 40.5$ months (range 6-191 months). As expected, imaging follow-up was longer in early GKRS group $(66.0 \pm 46.44$ months) than in the late GKRS group $(43.83 \pm 33.22$ months $)(\mathrm{p}=0.002)$. Only one patient treated before 2000 experienced tumor progression, and there was no documented tumor progression among patients treated in or after 2000.

In all, 47 patients (35\%) had some degree of a new pituitary deficiency at the last follow-up, and this rate was not different between the two groups. The incidence of new in estrogen/testosterone deficiency was higher in early than late GKRS patients $(22 \%$ vs $8 \%, \mathrm{p}=0.018)$. The incidence of new visual deficits $(n=2)$ and other cranial nerve deficits $(n=2)$ in the entire cohort was low and similar between the two treatment groups. The incidence of new visual deficits was greater in patients who received fractionated radiation therapy before GKRS versus those who did not (33\% vs $0 \%$, respectively; $p<0.001)$. This was not the case for new pituitary deficiency $(\mathrm{p}=0.334)$.

Thirteen patients (10\%) required a second GKRS treatment after the original procedure, and this rate was higher in the early rather than late GKRS group (16\% vs 5\%, respectively; $p=0.03$ ). The need for additional adenoma resection, adrenalectomy, or medical therapy was not related to the era of GKRS.

TABLE 3. Association of GKRS era with endocrine remission

\begin{tabular}{lccccc}
\hline & \multicolumn{2}{c}{ Endocrine Remission } & & \multicolumn{2}{c}{ Cox Regression: GKRS at $\geq 2000$ vs $<2000$} \\
\cline { 2 - 3 } \cline { 5 - 6 } Time After GKRS & GKRS $<2000$ & GKRS $\geq 2000$ & & Univariate $^{*}$ & Multivariate $^{*} \dagger$ \\
\hline 1 yr after GKRS & $16 \%$ & $44 \%$ & & $2.012(1.208-3.352), p=0.007$ & $2.259(1.219-4.186), p=0.01$ \\
\hline 2 yrs after GKRS & $38 \%$ & $63 \%$ & & $2.277(1.438-3.606), p<0.001$ & $2.501(1.448-4.320), p=0.001$ \\
\hline 5 yrs after GKRS & $64 \%$ & $82 \%$ & & $1.961(1.289-2.982), p=0.002$ & $2.021(1.249-3.268), p=0.004$ \\
\hline Entire follow-up & $66 \%$ & $82 \%$ & & $1.939(1.278-2.941), p=0.002$ & $1.987(1.234-3.199), p=0.005$ \\
\hline
\end{tabular}

* Values are presented as hazard ratios $(95 \% \mathrm{Cls})$, with the respective $p$ values.

$\dagger$ Adjusted for patient age, sex, pre-GKRS surgery, pre-GKRS fractionated radiation therapy, prescription dose, treatment volume, sellar treatment, suprasellar treatment, and cavernous sinus treatment. 
TABLE 4. Imaging outcomes and adverse events after GKRS

\begin{tabular}{|c|c|c|c|c|}
\hline \multirow[b]{2}{*}{ Imaging Outcomes \& Adverse Events } & \multirow[b]{2}{*}{ Total Sample } & \multicolumn{2}{|c|}{ Era of GKRS } & \multirow{2}{*}{$\begin{array}{c}\text { Chi-Square Test ( } p) \text { or ANOVA } \\
\text { F-Value }(p)\end{array}$} \\
\hline & & $<2000$ & $2000-2019$ & \\
\hline Imaging outcomes after GKRS & & & & $1.447(0.229)$ \\
\hline Stable & $133(99 \%)$ & $54(98 \%)$ & $79(100 \%)$ & \\
\hline Progression* & $1(1 \%)$ & $1(2 \%)$ & $0(0 \%)$ & \\
\hline Interval from GKRS to last MRI & $52.93 \pm 40.53$ & $66.0 \pm 46.44$ & $43.83 \pm 33.22$ & $10.392(0.002)$ \\
\hline \multicolumn{5}{|l|}{ New pituitary deficiency after GKRS } \\
\hline Any & $47(35 \%)$ & $21(38 \%)$ & $26(33 \%)$ & $0.396(0.529)$ \\
\hline Hypothyroidism & $30(22 \%)$ & $16(29 \%)$ & $14(18 \%)$ & $2.412(0.120)$ \\
\hline Estrogen/testosterone deficiency & $18(13 \%)$ & $12(22 \%)$ & $6(8 \%)$ & $5.614(0.018)$ \\
\hline Growth hormone deficiency & $23(17 \%)$ & $11(20 \%)$ & $12(15 \%)$ & $0.528(0.468)$ \\
\hline Diabetes insipidus & $3(2 \%)$ & $1(2 \%)$ & $2(3 \%)$ & $0.075(0.784)$ \\
\hline Hypocortisolism & $22(16 \%)$ & $8(15 \%)$ & $14(18 \%)$ & $0.238(0.625)$ \\
\hline New visual deficits after GKRS & $2(2 \%)$ & $2(4 \%)$ & $0(0 \%)$ & $2.916(0.09)$ \\
\hline New other $\mathrm{CN}$ deficits after GKRS & & & & $2.134(0.344)$ \\
\hline CN III & $1(1 \%)$ & $0(0 \%)$ & $1(1 \%)$ & \\
\hline Multiple & $1(1 \%)$ & $1(2 \%)$ & $0(0 \%)$ & \\
\hline Endocrine recurrence $†$ & 18/101 (18\%) & $13 / 36(36 \%)$ & $5 / 65(8 \%)$ & $12.776(<0.001)$ \\
\hline Time to endocrine recurrence, mos & $34.61 \pm 31.63$ & $36.46 \pm 35.79$ & $29.80 \pm 19.21$ & $0.152(0.702)$ \\
\hline \multicolumn{5}{|l|}{ Additional treatments after GKRS } \\
\hline Repeat GKRS & $13(10 \%)$ & $9(16 \%)$ & $4(5 \%)$ & $4.727(0.03)$ \\
\hline Pituitary adenoma surgery & $14(10 \%)$ & $8(15 \%)$ & $6(8 \%)$ & $1.674(0.196)$ \\
\hline Bilateral adrenalectomy & $14(10 \%)$ & $9(17 \%)$ & $5(6 \%)$ & $3.589(0.166)$ \\
\hline Fractionated radiotherapy & $0(0 \%)$ & $0(0 \%)$ & $0(0 \%)$ & NA \\
\hline Initiation of new medical therapy & $3(2 \%)$ & $0(0 \%)$ & $3(4 \%)$ & $2.136(0.144)$ \\
\hline
\end{tabular}

\section{Discussion}

To the best of our knowledge, this is the first study to evaluate the association of the era of GKRS treatment (as a proxy of technological GKRS advancements and center experience) with treatment outcomes. We found that the likelihood for endocrine remission was greater, time to endocrine remission was shorter, and the risk of endocrine recurrence was lower in patients treated with GKRS in 2000 and later than patients treated before 2000. Treatment volume, number of isocenters, prescription dose, maximal dose to the optic chiasm and frequency of cavernous sinus treatment were greater in the contemporary GKRS cohort than in the early GKRS cohort. Local tumor control rates and AREs rates were similar between the two groups.

The most important observation of our study is that endocrine remission results were better in the contemporary GKRS cohort (treated in 2000 or later) than in the early GKRS cohort (treated before 2000), suggesting that technological/procedural improvements and increasing clinical experience are important for GKRS results in cases of CD. The year 2000 was previously shown to be pivotal for outcomes with GKRS for arteriovenous malformations. ${ }^{24}$ These findings contribute to the growing body of evidence that the greater experiences of a neurosurgeon and center (i.e., the treatment team) are associated with better treatment results across a spectrum of neurosurgical procedures, including transsphenoidal resection of pituitary adenomas, ${ }^{4,16}$ excision of vestibular schwannomas, ${ }^{2,18}$ intracranial aneurysm clipping ${ }^{11}$ and coiling, ${ }^{1}$ and scoliosis correction surgery, ${ }^{8}$ among others. Similarly, studies from the radiation oncology literature also indicate that there are both institutional and individual learning curves for radiation therapy planning when treating locally advanced head and neck cancer ${ }^{17}$ and primary lung cancer. ${ }^{15}$ Our findings remain to be replicated in independent studies. The current studies suggest that there is a learning curve for GKRS procedures, and this should be considered during neurosurgical training and practice. Given the interdisciplinary nature of GKRS treatment planning and the often close proximity of GKRS radiosurgical targets (pituitary adenomas) to critical neurovascular structures, adequate training is imperative before starting independent practice and after major upgrades to GKRS devices. Continuous quality control and knowledge updates should be considered during independent practice to ensure optimal patient care.

Since its widespread introduction in clinical practice in the late 1980s until now, there have been numerous devices and software improvements in GKRS systems that advanced targeting precision, planning, delivery, and procedural workflow. For example, the first commercially available GKRS systems relied on relatively low numbers of isocenter targeting that were manually selected, and the 
accuracy of target identification was limited by the available images at that time (generally CT based). At present, however, contemporary GKRS systems allow multiple isocenter planning that results in more conformal dose plans, MRI-based planning for better target delineation, and composite isocenters for more elegant dose distribution and sparing of critical structures.

GKRS treatment planning parameters were different in early compared to the contemporary GKRS cohort. We observed that the treatment volume was larger in patients treated in 2000 and later, and this can be explained by a greater proportion of patients who had their cavernous sinuses treated for tumor infiltration and by a larger number of isocenters used, allowing for more conformal treatment target delineation. Improved imaging modalities allowed more reliable identification of residual adenoma tissue within the cavernous sinuses. Increasing neurosurgeon experience and better intraoperative visualization of residual and infiltrative adenoma at the time of microsurgery and/or radiosurgery allow the use of this information for GKRS planning purposes and more complete radiosurgical targeting of the adenoma. The group from the University of Pittsburgh also found that a greater number of isocenters was used for treatment with GK model $\mathrm{C}$ unit with an automatic positioning system when compared with model $\mathrm{U}$, which can result in a more conformal dose plan with newer GKRS technology. ${ }^{9}$ On the other hand, studies comparing Gamma Knife models 4C with the the Perfexion did not find significant differences in the number of isocenters used per treatment session and dose conformity. ${ }^{22,27}$ These findings illustrate that technological advances of GKRS systems, improved neuroimaging methods, and surgical tools have changed GKRS treatment planning. Therefore, GKRS treatment planning paradigms that were based on early experiences with GKRS systems may need to be revised and carefully reconsidered before applying them to treatment planning with contemporary Gamma Knife systems. Furthermore, historical treatment results of CD using early GKRS systems might not accurately predict treatment results with contemporary GKRS systems. Continuous updating of research outcomes is needed to better inform about optimal GKRS treatment strategies using contemporary GKRS system, and GKRS model/planning software should be carefully considered when reporting treatment results.

Imaging methods, surgical approaches to the sellar region, and intraoperative visualization have significantly evolved over the 30 years of the study. ${ }^{4}$ However, resection of adenomas was the preferred initial treatment option throughout the course of the study. The majority of CD patients (98\%) treated with GKRS at the University of Virginia underwent adenoma resection prior to GKRS. GKRS was considered in cases of incomplete adenoma resection or at the time of $\mathrm{CD}$ recurrence. On the other hand, medical treatment options and follow-up strategies for CD remained largely unchanged during the course of the study, and imaging of ACTH-producing pituitary adenomas can be challenging even using high- and ultrahigh-resolution MRI scans.

The incidence rates of cranial neuropathy and some degree of new anterior pituitary gland deficiency were similar between the two groups despite the greater mean radiation dose to the optic chiasm $(6.4 \pm 2.2 \mathrm{~Gy}$ in the contemporary cohort vs $3.3 \pm 2.3$ Gy in the early cohort), greater treatment volumes, and more common targeting of the cavernous sinus in the contemporary cohort. These findings can be attributed to increasing personal and published experiences regarding optic chiasm tolerance limits, advances in GKRS targeting capabilities, and imaging advances, allowing more confident and safe treatments of residual adenomas residing in close proximity to the anterior optic apparatus.

\section{Study Limitations and Strengths}

This study has limitations that should be acknowledged. First, the retrospective design and single-institution series are subject to selection bias. However, all patients were managed according to the prevailing guidelines at the time of treatment, and $98 \%$ of patients had prior attempts at adenoma resection and underwent GKRS for residual or recurrent disease. Second, during almost 4 decades of the study there have been numerous improvements in surgical techniques and imaging methods, which might have affected treatment results above and beyond treatment with GKRS, but they were not systematically accounted for in this series. Also, more subtle engineering/ software upgrades of GKRS systems might have affected the precision of planning and radiation delivery, and hence treatment results, but were not considered because of small sample sizes. Instead, we chose the GKRS treatment date cutoff at the year 2000 because, at that time, high-resolution MRI became the standard of care, and there were significant improvements in GKRS systems. Finally, our results cannot be extrapolated to patients treated with fractionated radiation therapy because we studied only patients treated with GKRS.

The strengths of this study include inclusion of consecutive patients treated with GKRS at a single institution since the installation of the first commercially available GKRS system until the present day, allowing us to evaluate the possible role of changing GKRS technology and other imaging/surgical improvements for treatment safety and efficacy while avoiding interinstitutional differences in treatment strategies.

\section{Conclusions}

Advancements of GKRS systems and a center's growing experience are associated with improved treatment outcomes of patients with CD. Local tumor control rates and ARE rates were similar in the early and contemporary cohorts. There has been a shift in treatment planning parameters over time. Technological aspects and results of contemporary GKRS systems should be considered when planning GKRS treatment and counseling patients. Further studies exploring a learning curve for GKRS should be considered, and such a learning curve for SRS should have implications for neurosurgical training.

\section{References}

1. Anderson IA, Kailaya-Vasan A, Nelson RJ, Tolias CM: Clipping aneurysms improves outcomes for patients undergoing coiling. J Neurosurg 130:1491-1497, 2019

2. Barker FG II, Carter BS, Ojemann RG, Jyung RW, Poe DS, 
McKenna MJ: Surgical excision of acoustic neuroma: patient outcome and provider caseload. Laryngoscope 113:13321343,2003

3. Clayton RN, Jones PW, Reulen RC, Stewart PM, HassanSmith ZK, Ntali G, et al: Mortality in patients with Cushing's disease more than 10 years after remission: a multicentre, multinational, retrospective cohort study. Lancet Diabetes Endocrinol 4:569-576, 2016

4. Fatemi N, Dusick JR, de Paiva Neto MA, Kelly DF: The endonasal microscopic approach for pituitary adenomas and other parasellar tumors: a 10-year experience. Neurosurgery 63 (4 Suppl 2):244-256, 2008

5. Feelders RA, Pulgar SJ, Kempel A, Pereira AM: The burden of Cushing's disease: clinical and health-related quality of life aspects. Eur J Endocrinol 167:311-326, 2012

6. Ganz JC: Changing the gamma knife. Prog Brain Res 215:117-125, 2014

7. Graversen D, Vestergaard P, Stochholm K, Gravholt CH, Jørgensen JOL: Mortality in Cushing's syndrome: a systematic review and meta-analysis. Eur J Intern Med 23:278-282, 2012

8. Hyun SJ, Han S, Kim KJ, Jahng TA, Kim YJ, Rhim SC, et al: Adolescent idiopathic scoliosis surgery by a neurosurgeon: learning curve for neurosurgeons. World Neurosurg 110:e129-e134, 2018

9. Kondziolka D, Maitz AH, Niranjan A, Flickinger JC, Lunsford LD: An evaluation of the Model C gamma knife with automatic patient positioning. Neurosurgery 50:429-432, 2002

10. Lambert JK, Goldberg L, Fayngold S, Kostadinov J, Post KD, Geer EB: Predictors of mortality and long-term outcomes in treated Cushing's disease: a study of 346 patients. J Clin Endocrinol Metab 98:1022-1030, 2013

11. Lawton MT, Du R: Effect of the neurosurgeon's surgical experience on outcomes from intraoperative aneurysmal rupture. Neurosurgery 57:9-15, 2005

12. Leksell L: The stereotaxic method and radiosurgery of the brain. Acta Chir Scand 102:316-319, 1951

13. Lindholm J, Juul S, Jørgensen JO, Astrup J, Bjerre P, FeldtRasmussen U, et al: Incidence and late prognosis of Cushing's syndrome: a population-based study. J Clin Endocrinol Metab 86:117-123, 2001

14. Lindquist C, Paddick I: The Leksell Gamma Knife Perfexion and comparisons with its predecessors. Neurosurgery 61 (3 Suppl):130-141, 2007

15. Lo AC, Liu M, Chan E, Lund C, Truong PT, Loewen S, et al: The impact of peer review of volume delineation in stereotactic body radiation therapy planning for primary lung cancer: a multicenter quality assurance study. J Thorac Oncol 9:527-533, 2014

16. Lofrese G, Vigo V, Rigante M, Grieco DL, Maresca M, Anile $\mathrm{C}$, et al: Learning curve of endoscopic pituitary surgery: experience of a neurosurgery/ENT collaboration. J Clin Neurosci 47:299-303, 2018

17. Maguire PD, Honaker G, Neal C, Meyerson M, Morris D, Rosenman J, et al: A bridge between academic and community radiation oncology treatment planning. J Oncol Pract 3:238-241, 2007

18. McClelland S III, Guo H, Okuyemi KS: Morbidity and mortality following acoustic neuroma excision in the United States: analysis of racial disparities during a decade in the radiosurgery era. Neuro Oncol 13:1252-1259, 2011

19. Mehta GU, Ding D, Patibandla MR, Kano H, Sisterson N, Su YH, et al: Stereotactic radiosurgery for Cushing disease: results of an international, multicenter study. J Clin Endocrinol Metab 102:4284-4291, 2017

20. Monaco EA, Grandhi R, Niranjan A, Lunsford LD: The past, present and future of Gamma Knife radiosurgery for brain tumors: the Pittsburgh experience. Expert Rev Neurother 12:437-445, 2012
21. Nieman LK, Biller BMK, Findling JW, Murad MH, NewellPrice J, Savage MO, et al: Treatment of Cushing's syndrome: an Endocrine Society clinical practice guideline. J Clin Endocrinol Metab 100:2807-2831, 2015

22. Niranjan A, Novotny J Jr, Bhatnagar J, Flickinger JC, Kondziolka D, Lunsford LD: Efficiency and dose planning comparisons between the Perfexion and 4C Leksell Gamma Knife units. Stereotact Funct Neurosurg 87:191-198, 2009

23. Oldfield EH: Cushing's disease: lessons learned from 1500 cases. Neurosurgery 64 (CN Suppl 1):27-36, 2017

24. Patibandla MR, Ding D, Kano H, Starke RM, Lee JYK, Mathieu D, et al: Effect of treatment period on outcomes after stereotactic radiosurgery for brain arteriovenous malformations: an international multicenter study. J Neurosurg 130:579-588, 2019

25. Pendharkar AV, Sussman ES, Ho AL, Hayden Gephart MG, Katznelson L: Cushing's disease: predicting long-term remission after surgical treatment. Neurosurg Focus 38(2):E13, 2015

26. Petersenn S, Beckers A, Ferone D, van der Lely A, Bollerslev J, Boscaro M, et al: Therapy of endocrine disease: outcomes in patients with Cushing's disease undergoing transsphenoidal surgery: systematic review assessing criteria used to define remission and recurrence. Eur J Endocrinol 172:R227R239, 2015

27. Régis J, Tamura M, Guillot C, Yomo S, Muraciolle X, Nagaje M, et al: Radiosurgery with the world's first fully robotized Leksell Gamma Knife PerfeXion in clinical use: a 200-patient prospective, randomized, controlled comparison with the Gamma Knife 4C. Neurosurgery 64:346-356, 2009

28. Sharma ST, Nieman LK, Feelders RA: Comorbidities in Cushing's disease. Pituitary 18:188-194, 2015

29. Sheehan J, Steiner L: A perspective on radiosurgery: creativity, elegance, simplicity, and flexibility to change. World Neurosurg 80:83-86, 2013

30. Snell JW, Sheehan J, Stroila M, Steiner L: Assessment of imaging studies used with radiosurgery: a volumetric algorithm and an estimation of its error. Technical note. J Neurosurg 104:157-162, 2006

31. Starke RM, Reames DL, Chen CJ, Laws ER, Jane JA Jr: Endoscopic transsphenoidal surgery for Cushing disease: techniques, outcomes, and predictors of remission. Neurosurgery 72:240-247, 2013

32. Wu A, Lindner G, Maitz AH, Kalend AM, Lunsford LD, Flickinger JC, et al: Physics of gamma knife approach on convergent beams in stereotactic radiosurgery. Int J Radiat Oncol Biol Phys 18:941-949, 1990

\section{Disclosures}

The authors report no conflict of interest concerning the materials or methods used in this study or the findings specified in this paper.

\section{Author Contributions}

Conception and design: J Sheehan. Acquisition of data: J Sheehan, D Sheehan, Schlesinger. Analysis and interpretation of data: J Sheehan, Bunevicius, Schlesinger. Drafting the article: Bunevicius. Critically revising the article: all authors. Reviewed submitted version of manuscript: all authors. Approved the final version of the manuscript on behalf of all authors: J Sheehan. Statistical analysis: Bunevicius. Administrative/technical/material support: J Sheehan. Study supervision: J Sheehan.

\section{Correspondence}

Jason P. Sheehan: University of Virginia Health System, Charlottesville, VA. jps2f@hscmail.mcc.virginia.edu. 